\section{Comment on "What is the Most General Abelian Gauge Theory in Two Spatial Dimensions?"}

In a recent Letter, ${ }^{1}$ Hagen has proposed a vector-field action in three space-time dimensions, and claimed to have found a new massless excitation in a particular limit. We note here that this action is essentially the one given earlier by us $^{2}$ to represent the Maxwell-ChernSimons system: His action differs from ours by an additional term which is inessential and by including an extra parameter which can likewise be rescaled away. The "new" excitation is shown to be just the photon.

We use the notation and (implicit) conventions of Ref. 1, whose Lagrangean is

$$
\begin{aligned}
L_{1}=-\frac{1}{2} \mu \Phi_{\mu}^{2}- & \frac{1}{2} \lambda \epsilon^{\mu \alpha v} \phi_{\mu} \partial_{\alpha} \phi_{v} \\
& -\epsilon^{\mu \alpha v} \Phi_{\mu} \partial_{\alpha} \phi_{v}-\frac{1}{2} \epsilon^{\mu \alpha v} \Phi_{\mu} \partial_{\alpha} \Phi_{v} .
\end{aligned}
$$

This is to be compared with that of Ref. 2,

$$
L_{2}=-\frac{1}{2} \Phi_{\mu}^{2}-\frac{1}{2} \bar{\lambda} \epsilon^{\mu \alpha v} \phi_{\mu} \partial_{\alpha} \phi_{\nu}-\epsilon^{\mu \alpha v} \Phi_{\mu} \partial_{\alpha} \phi_{\nu}
$$

The first three terms are identical, up to the parameter $\mu$ which (if not singular) can be rescaled by appropriate renaming of $\Phi, \phi$, and $\bar{\lambda}$. The fourth term in (1) is new.

Now it was shown in Ref. 2 that $L_{2}$ is just another form of the topologically massive action (see Deser, Jackiw, and Templeton ${ }^{3}$ for its analysis and earlier references) and that it is also equivalent to the "self-dual" form given by Townsend, Pilch, and van Nieuwenhuizen. ${ }^{4}$ In particular, then, the limits $\bar{\lambda}=0$ and $\bar{\lambda}=\infty$ in (2) correspond, respectively, to pure Maxwell and pure Chern-Simons theory. That the fourth term in (1) plays no additional role is easily understood from the fact that elimination of $\phi_{\mu}$ from (2) was shown to yield the "self-dual" form of Ref. 2. The fourth term in (1) is a "spectator" in this elimination, and merely changes the coefficient of one of the resulting two terms. Thus, it does not affect anything but the singular $\lambda=0$ case, and is superfluous to the description of the vector system.

In the singular case $\lambda=0,(1)$ is actually parity preserving and reduces to the standard antisymmetric tensor representation of a massless scalar ${ }^{5}$ upon translating $\phi_{v}$ by $\frac{1}{2} \Phi_{v}$ to remove the last term, then replacing it by its dual; this explains the author's finding that the system is massless. It is easy to show explicitly that this theory is, in fact, Maxwell's [again translate $\phi_{v}$ in (1), replace $\Phi_{\mu}$ by its dual, and scale $\mu$ away], but the following argument is perhaps more instructive. In $D=3 \mathrm{di}$ mensions, the photon is spinless ${ }^{3,6}$ because if it had spin 1 , it would have to have two "helicity" states (by parity), and it has but one degree of freedom here. Thus there is only one massless particle in $D=3$ dimensions, which can be expressed at will by a vector or scalar or higherorder tensor. Similarly, there are two possible massive spin-1 systems, the topologically massive parity-nonconserving action with one helicity, or its parity doublet extension, equivalent to the Proca massive-vector theory. Finally, there is the nondynamical pure Chern-Simons action. Any irreducible vector or scalar action therefore represents one of these systems: if massless and therefore parity preserving, the scalar/Maxwell theory (or Proca theory if $m \neq 0$ ); if parity nonconserving, the topologically massive (for $m \neq 0$ ) or pure Chern-Simons theory (for $m \rightarrow \infty$ ).

All the above results may be checked explicitly by canonical reduction of the respective actions to yield the stated particle contents. We also remark that the stress tensor corresponding to (1) is manifestly identical to that of (2) since none of the terms containing $\epsilon^{\mu \alpha v}$ contribute; this provides an independent means of showing equivalence.

Coupling to external sources will, of course, appear different in different representations; this was analyzed in Ref. 2 and incidentally provides yet another means of identifying the excitations. In this connection, it is stressed in Ref. 1 that external currents can couple to the (dual) field strength and claimed that this has novel consequences. However, direct current coupling to field strength is essentially equivalent to minimal coupling to a current which is the old one's curl, and we see no special significance in this possibility.

This work was supported in part by National Science Foundation Grant No. PHY-82-01094 and through funds provided by the U.S. Department of Energy under Contract No. DE-AC02-76ER03069.

S. Deser

Department of Physics

Brandeis University

Waltham, Massachusetts 02254

R. Jackiw

Department of Physics

Massachusetts Institute of Technology

Cambridge, Massachusetts 02139

Received 13 April 1987

PACS numbers: 11.15.-q, 11.10.Ef

${ }^{1}$ C. R. Hagen, Phys. Rev. Lett. 58, 1074 (1987).

${ }^{2}$ S. Deser and R. Jackiw, Phys. Lett. 139B, 371 (1984).

${ }^{3}$ S. Deser, R. Jackiw, and S. Templeton, Phys. Rev. Lett. 48, 975 (1982), and Ann. Phys. (N.Y.) 140, 372 (1982).

${ }^{4} \mathrm{P}$. K. Townsend, K. Pilch, and P. van Nieuwenhuizen, Phys. Lett. 136B, 38 (1984), and 137B, 443 (1984).

${ }^{5}$ V. I. Ogievetsky and I. V. Polubarinov, Yad. Fiz. 4, 216 (1966) [Sov. J. Nucl. Phys. 4, 156 (1967)]; S. Deser, Phys. Rev. 187, 1931 (1969).

${ }^{6}$ B. Binegar, J. Math. Phys. 23, 1511 (1982). 